SCIENTIFIC LETTER

\title{
Plasma homocysteine and calcific aortic valve disease
}

\author{
G M Novaro, H D Aronow, E Mayer-Sabik, B P Griffin
}

Heart 2004;90:802-803. doi: 10.1136/hrt.2003.022889

C alcific aortic valve disease and coronary atherosclerotic disease both demonstrate histological features of endothelial disruption and subendothelial accumulation of lipid, lipoprotein, and inflammatory cells. ${ }^{1}$ These entities also share acknowledged risk factors, ${ }^{2}$ including hypercholesterolaemia, hypertension, and diabetes mellitus, believed to cause disease at least partly via endothelial injury. Data suggest that raised homocysteine concentrations, also associated with endothelial damage, ${ }^{3}$ may be an independent risk factor for coronary artery disease. ${ }^{4}$ Whether raised homocysteine is a risk factor for calcific aortic valve disease is unknown. We hypothesised that increasing homocysteine concentrations might predict the prevalence of calcific aortic valve disease, specifically aortic sclerosis and aortic stenosis, in a group of patients undergoing cardiac surgery.

\section{METHODS}

We retrospectively analysed 76 patients with a mean (SD) age of 66 (13) years, (65\% male) who underwent cardiac surgery at the Cleveland Clinic Foundation with intraoperative transoesophageal echocardiography. The patients were originally recruited for a prospective study assessing the relation between plasma homocysteine and aortic atheroma. Exclusion criteria were chronic renal failure, rheumatic heart disease, and known malignancy. All subjects gave informed consent and our institutional review board approved the study. All patients had fasting plasma homocysteine concentrations obtained on the day of their preoperative evaluation or on the morning of surgery. Venous blood was placed into a heparinised tube on ice, centrifuged within 10 minutes of blood being drawn, and analysed using a microparticle enzyme immunoassay. Normal plasma homocysteine ranges in our laboratory are 6.4-13.7 $\mu \mathrm{mol} / \mathrm{l}$ for men and 3.4-12.9 $\mu \mathrm{mol} / \mathrm{l}$ for women.

The following baseline clinical characteristics were recorded: age, sex, serum creatinine concentration, and history of hypertension, hypercholesterolaemia, tobacco use, and diabetes mellitus. All patients underwent intraoperative transoesophageal echocardiography at the time of surgery with 5.0 MHz biplane or multiplane probes. The aortic valve was coded as normal, aortic sclerosis (thickening and calcification of aortic leaflets without left ventricular outflow obstruction) or aortic stenosis (thickening and calcification of aortic leaflets, impaired systolic mobility, and a mean transvalvar gradient $>10 \mathrm{~mm} \mathrm{Hg}$ ). A staff echocardiographer, blinded to homocysteine concentrations, ascertained the degree of aortic valve disease. Continuous variables are expressed as medians with interquartile ranges (IQR). Categorical data appear as frequencies and percentages. Kruskal-Wallis tests were utilised for bivariate analyses of continuous data. $\chi^{2}$ Tests were employed for bivariate analyses of categorical data.

\section{RESULTS}

Clinical characteristics appear in table 1. Median (IQR) homocysteine concentration was 11.7 (8.8-15.2) $\mu \mathrm{mol} / \mathrm{l}$. In bivariate analyses, plasma homocysteine concentrations were significantly higher with worsening aortic valve disease (table 1). Other bivariate predictors included age, hypercholesterolaemia, and serum creatinine. No association was found between homocysteine concentrations and sex, smoking, or hypertension. In multivariate analysis, after adjusting for age, hypercholesterolaemia, and serum creatinine, increasing plasma homocysteine was not associated with aortic sclerosis $(p=0.40)$ or stenosis $(p=0.25)$. Increasing age $(p=0.002)$ and serum creatinine $(p<0.001)$, however, remained significant independent predictors of aortic valve disease.

When comparing patients with aortic stenosis to those without stenotic aortic valves (that is, normal aortic valve or aortic sclerosis, $\mathrm{n}=59$ ), plasma homocysteine concentration was significantly higher in those with aortic stenosis compared to those without aortic stenosis (10.4 (8.5-12.9) $\mathrm{v}$ $16.6(12.7-17.8) \mu \mathrm{mol} / \mathrm{l}, \mathrm{p}=0.001)$. Patients with aortic stenosis, on average, were older $(p<0.001)$ and had higher serum creatinine concentrations $(p=0.008)$. In multiple logistic regression models, however, plasma homocysteine concentrations failed to predict aortic stenosis $(p=0.28)$, whereas increasing age remained a significant predictor $(\mathrm{p}<0.001)$.

Table 1 Clinical and laboratory characteristics

\begin{tabular}{lllll}
\hline & $\begin{array}{l}\text { Normal } \\
\text { valve(n=27) }\end{array}$ & $\begin{array}{l}\text { Aortic sclerosis } \\
(\mathbf{n = 3 2 )}\end{array}$ & $\begin{array}{l}\text { Aortic stenosis } \\
(\mathbf{n = 1 7})\end{array}$ & p Value \\
\hline Age (years) & $57(49-70)$ & $68(60-74)$ & $78(71-83)$ & $<0.001$ \\
Female (\%) & 30 & 34 & 47 & 0.49 \\
Hypertension (\%) & 52 & 50 & 53 & 0.98 \\
Hypercholesterolaemia (\%) & 48 & 81 & 41 & 0.006 \\
Diabetes mellitus (\%) & 22 & 28 & 12 & 0.42 \\
Tobacco use (\%) & 63 & 56 & 47 & 0.58 \\
Serum creatinine concentration & $0.9(0.8-1)$ & $1.0(0.9-1.2)$ & $1.2(1-1.3)$ & 0.004 \\
(mg/dl) & $9.2(8.2-14.6)$ & $11.3(9.3-12.8)$ & $16.6(12.7-17.8)$ & 0.003 \\
Plasma homocysteine & & & \\
concentration ( $\mu$ mol/l) & & & \\
\hline Continuous variables are expressed as medians (interquartile ranges) &
\end{tabular}




\section{DISCUSSION}

Our study was limited by its relatively small sample size. Although sufficiently large and powered to identify a continuous association between homocysteine concentrations and aortic valve disease, this study was not powered to detect a threshold effect, if one were present. In addition, our surgical patient population and cross sectional design limits its application to the larger general population.

Evidence now supports the notion that calcific aortic valve disease is the product of an active inflammatory process associated with a number of atherosclerotic risk factors. Although the damaging endothelial effects of homocysteine would suggest that raised concentrations of plasma homocysteine could initiate or facilitate the subendothelial inflammatory process responsible for calcific aortic valve disease, we found no evidence to support this hypothesis. To our knowledge, this is the first investigation exploring the potential association between homocysteine concentrations and calcific aortic valve disease. A previous study by Chan and colleagues assessed the relation between homocysteine and congenital bicuspid aortic stenosis, and also reported no significant association. ${ }^{5}$ Prior studies have demonstrated that plasma homocysteine concentrations increase with age and serum creatinine concentration. Similarly, we observed a correlation between increasing age and creatinine and homocysteine. It is conceivable that higher homocysteine concentrations seen in patients with renal impairment or older age might contribute to the greater prevalence and/or more rapid progression of aortic stenosis observed in these patient populations. A much larger analysis would be required to determine whether homocysteine were an intermediary step in the causal pathway between these risk factors and calcific aortic valve disease.

\section{Authors' affiliations}

G M Novaro, Department of Cardiology, Cleveland Clinic Florida, Weston, Florida, USA

H D Aronow, Division of Cardiovascular Medicine, University of Pennsylvania School of Medicine, Philadelphia, Pennsylvania, USA E Mayer-Sabik, B P Griffin, Department of Cardiovascular Medicine, Cleveland Clinic Foundation, Cleveland, Ohio, USA

Correspondence to: Dr Gian M Novaro, Department of Cardiology, Desk A23, Cleveland Clinic Florida, 2950 Cleveland Clinic Boulevard, Weston, FL 33331, USA; novarog@ccf.org

Accepted 13 October 2003

\section{REFERENCES}

1 Otto CM, Kuusisto J, Reichenbach DD, et al. Characterization of the early lesion of 'degenerative' valvular aortic stenosis. Histological and immunohistochemical studies. Circulation 1994;90:844-53.

2 Stewart BF, Siscovick D, Lind BK, et al. Clinical factors associated with calcific aortic valve disease. Cardiovascular health study. J Am Coll Cardiol 1997;29:630-4.

3 McCully KS. Vascular pathology of homocysteinemia: implications for the pathogenesis of arteriosclerosis. Am J Pathol 1969;56:111-28.

4 Clarke R, Daly L, Robinson K, et al. Hyperhomocystinemia: an independent risk factor for vascular disease. N Engl J Med 1991:324:1149-55.

5 Chan KL, Ghani M, Woodend K, et al. Case-controlled study to assess risk factors for aortic stenosis in congenitally bicuspid aortic valve. Am J Cardiol $2001 ; 88: 690-3$.

\section{IMAGES IN CARDIOLOGY}

\section{A novel streamlined "anchored" anatomical approach to ablation of AVNRT}

A practical approach to slow pathway ablation for the treatment of atrioventricular nodal re-entrant tachycardia (AVNRT) is to use a combined anatomic and electrogram technique to identify target sites. The efficacy and safety of radiofrequency (RF) ablation depend on the stability of the ablation catheter during RF energy delivery. Unusual anatomy, abrupt rhythm changes during RF application, and intraprocedural catheter and patient movement can pose significant challenges. Inadvertent AV block remains a concern occurring in approximately $1 \%$ of cases.

A 42 year old woman with a narrow complex regular tachycardia that had a pseudo $\mathrm{r}$ in Vl during tachycardia but not during sinus rhythm underwent a diagnostic electrophysiological study followed by RF ablation for cure of her AVNRT. The patient's own anatomy was leveraged not only as a means to localise an ablation target, but most importantly, as a stabilising factor during performance of slow pathway ablation. This highly streamlined approach can be performed safely, successfully, and rapidly entirely from the left femoral vein, through a single sheath, and with only two catheters in appropriately selected supraventricular tachycardia (SVT) patients.

A 12 French long sheath was introduced into the femoral vein and guided fluoroscopically to the base of the right atrium. A 6 French Bard EP-XT catheter was inserted into the coronary sinus (CS). Next, a 6 French EPT Blazer II ablation catheter was directed to the His and then, after confirming the diagnosis of AVNRT, moved to the putative anatomic region of the slow pathway. During three RF applications, the mobility constraints of the "sheath-catheter-catheter" complex led to excellent electrogram and ablation catheter

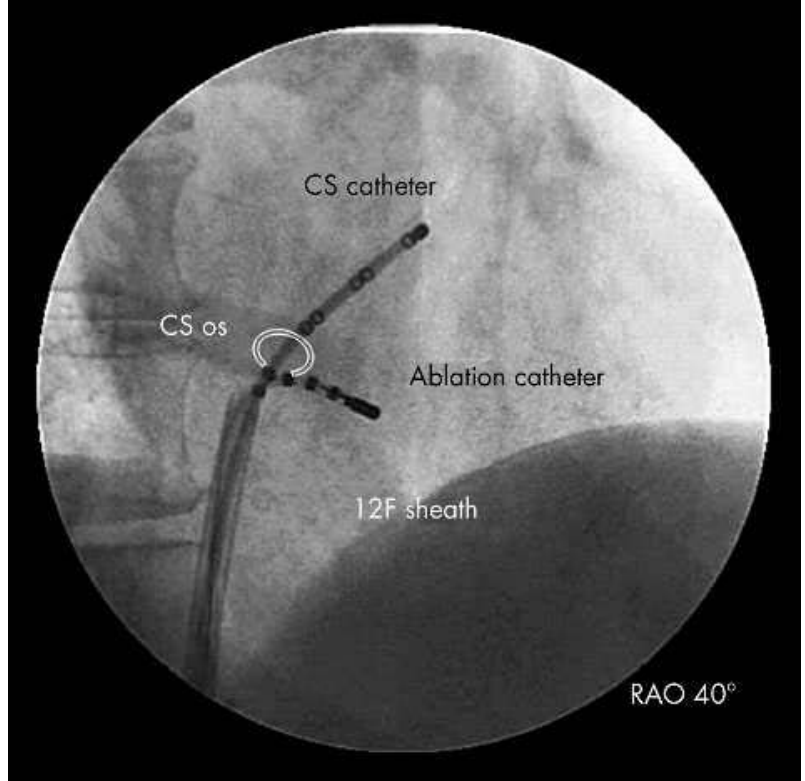

stability by taking advantage of the "collaring" effect of the 12 French sheath which was in turn "anchored" to the diagnostic catheter placed in the CS. No further tachycardia was inducible or has occurred clinically in follow up.

J R Gimbel gimbelir@ix.netcom.com 